\title{
Alternative Sources of Information and the Selection Decision Making Process
}

\author{
Bart L. Weathington \& Autumn R. Bechtel
}

The University of Tennessee at Chattanooga

\begin{abstract}
Author note
Correspondence related to this article should be addressed to Bart L. Weathington, University of Tennessee at Chattanooga, Department of Psychology, Dept. 2803, 615 McCallie Ave., Chattanooga, TN 37403-2598.

Contact: Bart-Weathington@utc.edu
\end{abstract}

\begin{abstract}
Personal information available on networking websites such as and Facebook ${ }^{\odot}$ and Linkedln ${ }^{\odot}$ is being used more frequently in the workplace. This study examined the effect of alternative sources of information (i.e., information beyond that provided on a formal resume) on applicant evaluations and selection decision making. Alternative information was provided in a format similar to that utilized on social networking websites. Specifically, information was provided about job candidates' alcohol consumption and gambling habits, information not typically found on resumes. It was hypothesized that evaluations of job candidates would be negatively influenced by the provision of negative information through a non resume source. The results indicated that alcohol consumption negatively affected evaluations of job candidates in five areas (likelihood of extending an interview invitation, assessment of qualifications, recommendation for hire, expected work performance, and expected work tenure). Gambling was found to negatively affect evaluations of job candidates in one area (expected work tenure), and the interaction between alcohol consumption and gambling was found to negatively affect evaluations of job candidates in three areas (assessment of qualifications, recommendation for hire, and expected work tenure). Consistent with the hypothesis, it appears that exposure to negative information through a non resume source negatively affected evaluations of job candidates.
\end{abstract}

Key Words: Employee Selection, Employee Discrimination, Gambling, Social Networking

\section{Introduction}

Personal information increasingly available on networking websites such as Facebook ${ }^{\odot}$ and Linkedln ${ }^{\odot}$ is being accessed more frequently in the workplace. Reports suggest 
employers are using this information for unintended (at least by applicants and employees) purposes such as selection and promotion decisions. The media have reported several cases of individuals losing jobs or not being hired due to information self-reported and posted on personal web pages (Finder, 2006; Mallory, 2007). The argument can be made that information available on the Internet should not be used for personnel decisions because much of it is proscribed for such purposes by Federal and State law (e.g., age, gender, religion, race, etc.). Further, the accuracy and validity of information found on personal web pages and Google ${ }^{\odot}$ information searches is of unknown quality and may be impossible to verify. It is technically feasible to create false web pages as personal attacks and practical jokes. In addition, online information searches can be misleading and inaccurate. Despite these concerns, however, this information is easily available and may impact professional decisions.

Information available via the internet differs from traditional sources of information. For example, the first impression many organizations have of applicants for professional positions is from their resumes (Gatewood \& Feild, 2001). While resumes are subject to errors and the falsification of information, it is generally possible to verify crucial information through the use of background checks and references. These options may not be viable for information obtained via non-traditional sources.

\section{Evaluating Applicants}

Hecker (1992) and Shelley $(1992,1994)$ anticipated that the number of jobs requiring a college degree in the 1990s and the early 2000s would not keep pace with the number of college graduates. According to Barton (2008), however, the rise in college attendance, coupled with downsizing, outsourcing, and a shortage of highpaying jobs is responsible for the increasing numbers of individuals with college degrees who do not earn above the national poverty level. Approximately 40 percent of college graduates are employed in a job where a college education is not required, in part because the economy has been forced to absorb the college graduates of the baby boom generation. In addition, fewer young college graduates have been able to obtain college labor market jobs (Barton, 2008). When such a gap exists between the number of college graduates and the number of available jobs requiring a college degree, job search skills become crucial (Occupational Outlook Quarterly, 1994). This is especially true about the preparation of an effective resume because companies depend heavily on resumes for hiring entry-level professional positions (Hutchinson, 1984) and a resume is the first major interaction between a job candidate and an organization (Thoms, McMaster, Roberts, \& Dombkowski, 1999).

Very few studies have been conducted on the validity of the inferences employers make in judging job applicants' skills and abilities from their resumes (Gatewood \& Field, 2001). However, it can be argued that more accurate and verifiable information will result in better inferences being made based on that information. Brown and Campion (1994) obtained ratings from recruiters on job applicant abilities and skills (language ability, math ability, physical abilities, interpersonal skills, leadership ability, and motivation) based on resume information. Regardless of the job, resume items were 
used by recruiters to infer job applicant attributes and moderate interrater reliability (.60s to .70s) was found for inferences of job applicant attributes. Recruiters inferred language and mathematics abilities from items related to educational achievement; physical abilities were judged from athletics or sports participation items; leadership and interpersonal attributes were seen from items displaying authority positions held and participation in social activities. Recruiters' inferences were believed to have face validity; however, there was little evidence regarding the empirical validity of recruiters' inferences from resume information (Brown \& Campion, 1994).

Thoms et al. (1999) used a policy-capturing experimental design to examine the impact of resume characteristics on decisions to interview recent college graduates. They developed seven sets of four resumes. The content (e.g., education, work experience) of all four resumes in each set was essentially identical. Participants were asked to select two out of four job candidates in each set that they would invite to a first screening interview and to include a one-sentence reason why selected candidates were chosen. Each set of resumes tested a different hypothesis and presented dichotomous choices.

Thoms et al. (1999) found that one page resumes lead to more interview invitations than two page resumes. In addition, resumes with general objective statements resulted in slightly more interview invitations than resumes that did not include general objective statements. For example, a general objective statement would include "Seeking a challenging professional position in a dynamic company with opportunities for development." Resumes with specific objective statements resulted in more interview invitations than resumes with general objective statements. Resumes with relevant coursework listed resulted in more interview invitations than resumes with no coursework listed. Resumes reporting high grade point averages resulted in more interview invitations than resumes that did not include or reported lower grade point averages. Lastly, resumes with accomplishment statements resulted in more interview invitations than resumes that did not include accomplishment statements. Taken together, Thoms et al.'s results suggest that certain resume characteristics may give applicants competitive advantages in obtaining interviews for first professional positions.

\section{$\underline{\text { Resume Biodata Information and Job Applicant Attributes }}$}

The employment interview is the most frequently employed selection technique (e.g., Arvey, 1979). However, the impact of the biographical information provided on resumes before and during the interview process is often overlooked (Dipboye, Fontenelle, \& Garner, 1984; Levine \& Flory, 1975; Pannone, 1994). Resumes provide employers a convenient and cost-effective method of assessing applicants' qualifications in areas such as education, work experience, and special skills (Cole, Field, \& Giles, 2003). Cole et al. (2003) examined whether recruiters' judgments of the presence of information reported on applicants' resumes was related to applicants' general mental ability and the Big Five personality dimensions. The main purpose was to determine the relationships between resume biodata information and applicant attributes. Specifically, Cole et al. (2003) examined whether recruiters' ratings of the presence of specific 
resume biodata was associated with six, job-relevant attributes: general mental ability and the Big Five personality dimensions.

Cole et al. (2003) found that there were many resume biodata items significantly associated with applicants' traits. Grade point averages were found to be positively related to applicants' conscientiousness scores. Individuals high in conscientiousness (characterized as purposeful, determined, and reliable) were probably able to better manage their academic responsibilities and attain more academic and professional achievements. They also found that listing relevant coursework on resumes had a negative relationship with extraversion. The resume biodata item, "has supervised others," was found to have a negative relationship with both agreeableness and conscientiousness. "Held a summer internship," was found to have a positive relationship with agreeableness and conscientiousness. "Honors/extracurricular activities" had the greatest number of significant relationships in the analyses. "Membership in professional societies" was found to have a positive relationship with conscientiousness. "Membership in college clubs and social fraternities/sororities" was found to have positive relationships with conscientiousness and extraversion and was also found to have a negative relationship with neuroticism. "Elected offices held," was found to have a negative relationship with neuroticism and a positive relationship with extraversion. Lastly, "received scholastic awards" was found to have positive relationships with both general mental ability and conscientiousness.

Cole et al. (2003) discovered differences among resume topics in terms of their effectiveness for predicting mental ability and the Big Five personality factors. Being a member of college clubs and in a social fraternity/sorority was found to have the greatest number of significant relationships with the six individual difference measures, whereas "supervised others," "held a summer internship," and "received scholastic awards" each had two significant relationships. In terms of the extent to which mental ability and the Big Five factors correlated with the resume items, conscientiousness had relationships with nine resume items, extraversion with seven, general mental ability with six, neuroticism with five, agreeableness with four, and openness to experience had only one relationship.

In sum, Cole et al. (2003) found that certain resume characteristics give job applicants competitive advantages in obtaining interviews. In addition, relationships exist between resume biodata and known predictors of job performance (e.g., general mental ability, the Big Five personality dimensions).

\section{Social Networking Websites}

In addition to traditional resumes, social networking websites are beginning to affect the selection decision making process (Finder, 2006).A social networking website is a place where individuals can communicate with friends, family, and meet new people on the Internet (Hargittai, 2008). Social networking websites allow individuals to share information, pictures, e-mails, and comments. For example, most MySpace ${ }^{\circledR}$ and Facebook $^{\odot}$ social networking websites include personal pictures and personal 
information (e.g., name, age, location, relationship status, religion, gender, race, hometown, sexual orientation, interests). Social networking websites have become some of the most popular online destinations in recent years.

\section{Employers and Social Networking Websites}

Finder (2006) described a situation where a small consulting company located in Chicago was looking for a summer intern. The company's president went on the Internet to check on a particularly promising job candidate who had just graduated from college. During the search, the executive found that the job candidate's interests included smoking marijuana, shooting people, and obsessive sex described in vivid detail. Having discovered this information, the company's president was hesitant to further interview this job candidate, questioning the job candidate's judgment (Finder, 2006).

Many companies that recruit on university campuses conduct background checks using search engines like Google ${ }^{\circledR}$ or Yahoo $^{\circledR}$ (Finder, 2006). College recruiters have admitted to looking up potential job applicants on social networking websites like Facebook ${ }^{\circledR}$, Myspace $^{\circledR}$, Xanga $^{\odot}$, and Friendster ${ }^{\odot}$. College students sometimes post questionable photographs and provocative comments about their alcohol consumption, recreational drug use, and sexual exploits. Recruiters have reported that some potential student job candidates appear immature and unprofessional on their personal web pages on social networking websites. College recruiters look for "red flags" or anything questionable that might go against their company's core values (Finder, 2006).

Companies can gain access to information on social networking websites in several ways (Finder, 2006). Recent graduates often keep their college e-mail addresses, which allow them to view some personal web pages. In addition, some companies ask college students working as interns to perform online background checks on job applicants. Microsoft stated that researching job candidates through social networking websites has become fairly typical. For the first time in history public information has become available about almost any job candidate. Correspondingly, college recruiters have begun warning students and providing cautionary tales about incidents involving personal web pages, online writing, and questionable photographs that raise serious doubts and questions from employers about job candidates' judgment (Finder, 2006).

Nathan Jones was a student from Penn State University who dressed up in a Virginia Tech shooting victim costume for Halloween 2007 and then posted pictures from the party on his Facebook ${ }^{\circledR}$ personal web page (Mallory, 2007). His Facebook ${ }^{\circledR}$ personal web page soon received national media coverage. In an e-mail he was formally asked to resign from his position at Bank of America or be fired because they did not want to be associated with the resulting scandal. Mr. Jones was forced to resign from Bank of America based on behavior that was documented and posted on Facebook ${ }^{\circledR}$ (Mallory, 2007).

The current study was designed to examine the effects of alternative sources of information on the selection decision making process. Alternative information was 
provided in a format similar to MySpace ${ }^{\odot}$ or Facebook ${ }^{\odot}$ personal web pages. This study also examined how job candidates' alcohol consumption and gambling habits impact the selection decision making process. Alcohol consumption and gambling habits were examined because these behaviors often appear as "red flags" to job candidates' dependability and trustworthiness.

In light of the reviewed literature, the following hypothesis is proposed: Hypothesis: Evaluations of job candidates will be negatively influenced by the provision of negative information through a non resume source.

\section{Method}

\section{Participants}

Participants in this study included 130 undergraduate and graduate students at a midsized university in the southeastern United States. The average age of participants was $24.88(S D=6.93)$, and the range of ages was 18 to 55 . Of the participants, 89 $(68.5 \%)$ were female and $41(31.5 \%)$ were male. Participants included $101(77.7 \%)$ Caucasian students, 20 (15.4\%) African American students, 1 (.8\%) Latino/Hispanic student, 4 (3.1\%) Asian/Asian American students, 1 (.8\%) Arab student, 1 (.8\%) Indian student, $1(.8 \%)$ black/French student, and $1(.8 \%)$ student who did not report race. Fifteen (11.5\%) participants were unemployed, $13(10 \%)$ participants were currently unemployed but had previously been employed, $65(50 \%)$ participants were employed part-time, $36(27.7 \%)$ participants were employed full-time, and $1(.8 \%)$ participant was seasonally employed. Participants included $5(3.8 \%)$ freshmen, $10(7.7 \%)$ sophomores, $28(21.5 \%)$ juniors, $38(29.2 \%)$ seniors, $48(36.9 \%)$ graduate students, and $1(.8 \%)$ returning student. The average personal annual income of participants was $\$ 15,615.48$ $(S D=\$ 18,408.84)$. The average family annual income of participants was $\$ 66,465.12$ $(\mathrm{SD}=\$ 55,745.72)$.

\section{Materials}

Participants were given a paper and pencil questionnaire packet. The questionnaire included an informed consent form, a demographic information form, an account administrator job description, a list of account administrator's job requirements, six different job candidates' resumes, six different job candidates' personal web pages, resume evaluation rating forms, and a overall hiring factors rating form.

The account administrator job description and job requirements list was provided by a financial services company located in Houston, Texas. Participants used the job description and job requirements list as a rating standard.

A set of six fictitious resumes were created: all male. All resumes were based on actual resumes submitted for an opening at the same financial services firm that provided the job description and job requirements. For ethical reasons and to preserve anonymity all resumes were modified to include fictitious addresses, names, and past employer 
information. Resume quality was controlled by utilizing the actual employer ratings of resumes from a real life hiring process. Over 100 resumes were received in response to the job posting and the six used in this study were the company's top six rated candidates for the job. All were judged to be equal by the human resource professionals responsible for hiring for the open position. Resumes reflected an occupational background in finance and/or accounting. Each job candidate was qualified for the available account administrator position and job candidates' resumes reflected job candidates' qualifications.

Personal web pages were created for each of the six job candidates to provide supplemental information. This information included job candidates' full name, picture, relationship status, sexual orientation, hometown, body type, ethnicity, zodiac sign, future plans for children, education level, smoking preference, general interests, music interests, movies interests, television interests, books interests, heroes, alcohol consumption, and gambling habits. All pictures were evaluated as equivalent in terms of attractiveness, perceived age, and likeability in a pilot test. Additionally, only pictures of white males were used. The researcher manipulated alcohol consumption and gambling habits. All other information was kept equivalent (i.e., all participants indicated they were heterosexual, did not smoke or have kids, etc.) and presented in a standard order/format. Profile information indicated that job candidates either drank a lot, some, or not at all and gambling interests were either included or not included in job candidates' general interests and web page graphics. One of the six possible combinations of alcohol use and gambling was represented on each web page.

\section{Design and Procedure}

Participants were selected through convenience sampling of undergraduate and graduate classes. Data was collected during class and required approximately 30 minutes to complete. To protect anonymity, the research packet did not require any personal identifying information to be provided. The questionnaire included a set of six resumes and a set of six personal web pages followed by a series of questions asking the participant to make employment decisions and recommendations that included assessments of recommendations to interview, ability, recommendations to hire, performance, and likelihood of quitting. Work-related skills were assessed using a 7point Likert-type scale.

Participants first read the informed consent form and then read over an account administrator job description and a job requirements list. Next, participants evaluated each job candidate's resume individually on the provided resume evaluation rating forms. Participants rated job candidates' resumes on five different criteria on a scale of one to seven. These five different areas of criteria addressed recommendations to interview, ability, recommendations to hire, performance, and likelihood of quitting.

Recommendations to Interview - Participants indicated their recommendations to interview each of the candidates on a 7 point Likert-type scale ( 1 = Strongly Disagree, 7 = Strongly Agree) in response to the following: "I recommend interviewing this 
applicant."

Ability - Participants were asked to indicate the perceived ability of each of the six candidates using a single item on a 7 point Likert-type scale $(1=$ Strongly Disagree, $7=$ Strongly Agree): "[Robert Parker] is highly qualified for this position."

Recommendations to Hire - Participants indicated their recommendations to hire each of the candidates on a 7 point Likert-type scale $(1=$ Strongly Disagree, $7=$ Strongly Agree) in response to the following: "I recommend hiring [Robert Parker] for this position."

Performance - Participants indicated their assessment of each candidate's anticipated performance with a single-item, 7 point Likert-type scale $(1=$ Poor, $7=$ Good $)$ in response to the following: "I expect [Robert Parker's] performance to be...."

Likelihood of Quitting - Participants indicated their assessment of each candidates' likelihood of quitting with a single-item, 7 point Likert-type scale $(1=$ Not Very Likely, $7=$ Very Likely) in response to the following: "The likelihood of [Robert Parker] quitting this job is..."

After initial evaluations were made, participants evaluated a paper copy of each job candidate's personal web page and completed a separate rating form for each. This information was provided based on the scenario that participants were hiring managers and their assistants, on their own, looked up the candidates online and found additional information on them. Participants then evaluated each job candidate again against the same criteria utilizing the same procedure. The only difference being that participants were asked to evaluate candidates based on all of the information now available to them - resumes and online profiles.

\section{Results}

Means and standard deviations for all variables and conditions are presented in Table 1. A two-way within-subjects analysis of variance (ANOVA) was conducted to evaluate changes in the evaluations of applicants from a resume-only condition to a resume plus web page condition when information about alcohol consumption and gambling habits was provided via a non-traditional (e.g., non resume) source. The dependent variables were changes in the individual job candidates' evaluation ratings on five criteria (likelihood of extending an interview invitation, assessment of qualifications, recommendation for hire, expected work performance, and likelihood of quitting). The within-subjects factors were gambling habits with two levels (gambling or no gambling) and alcohol consumption with three levels (a lot, some, no). The Alcohol Consumption main effect and Gambling Habits by Alcohol Consumption interaction effect was tested using repeated measures analysis of variance, based on multivariate tests of significance. 
Table 1

Descriptive Statistics

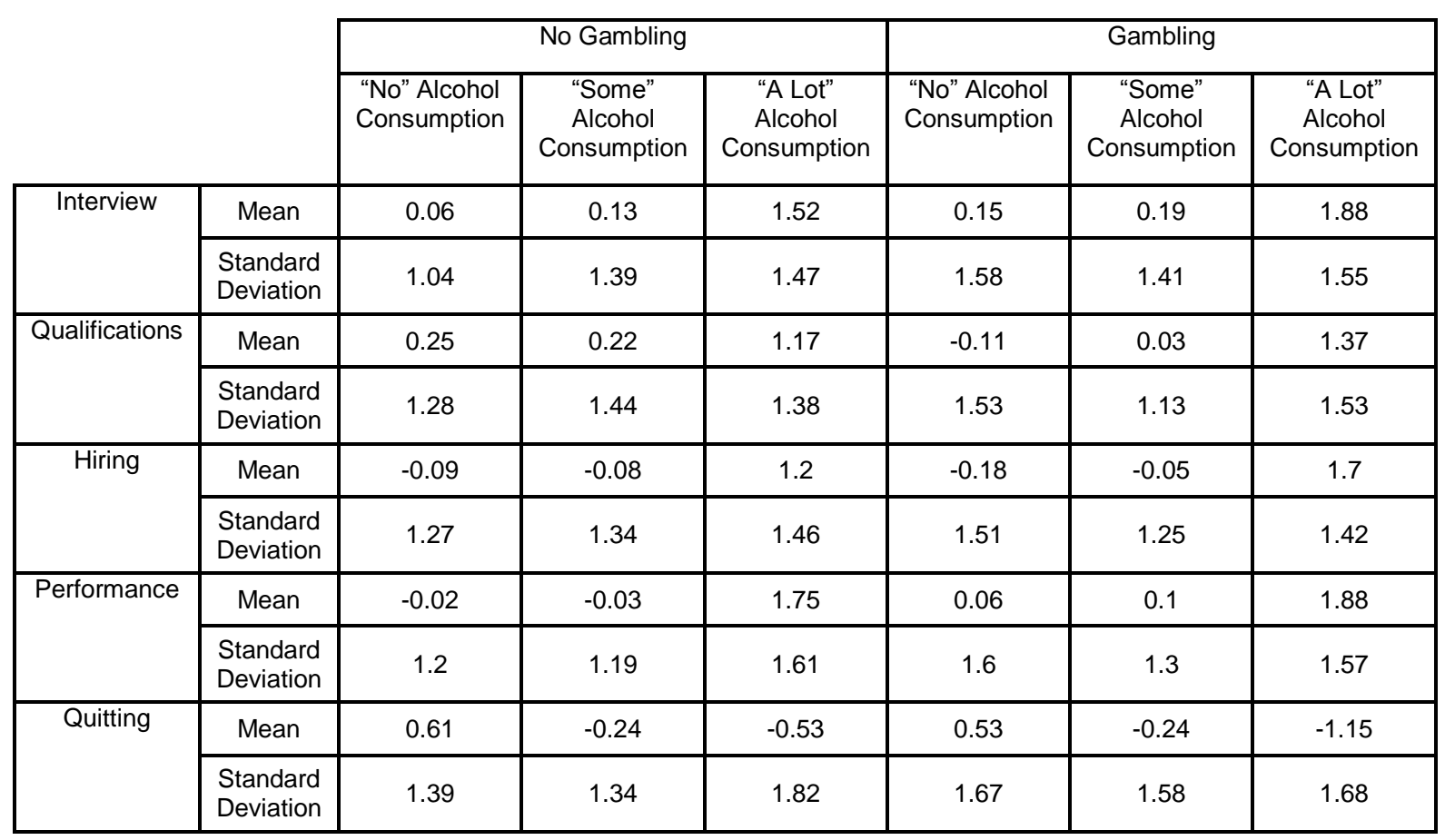

In regards to the likelihood of recommending job applicants interviews, a main effect of alcohol consumption was found to be significant, $F(2,127)=80.42, p<.001$, Partial Eta Squared $=.56$. Gambling was not found to be significant, $F(1,128)=3.16, p>.05$, Partial Eta Squared $=.02$. The interaction between alcohol consumption and gambling was not found to be significant, $F(2,127)=1.3, p>.05$, Partial Eta Squared $=.02$. A main effect of alcohol consumption was found to be significant. The higher job candidates' alcohol consumption level, the lower participants rated job candidates. Exposure to higher levels of alcohol consumption resulted in lower evaluation ratings.

In regards to the assessment of qualifications for the account administrator position, a main effect of alcohol consumption was found to be significant, $F(2,128)=68.64, p<$ .001 , Partial Eta Squared $=.52$. Gambling was not found to be significant, $F(1,129)=$ $1.73, p>.05$, Partial Eta Squared $=.01$. The interaction between alcohol consumption and gambling was found to be significant, $F(2,128)=3.59, p<.05$, Partial Eta Squared $=.05$, however, this significant interaction was driven by alcohol consumption. A main effect of alcohol consumption was found to be significant. The higher job candidates' alcohol consumption level, the lower participants rated job candidates. Exposure to higher levels of alcohol consumption resulted in lower evaluation ratings. The interaction between alcohol consumption and gambling habits was also found to be significant. Exposure to higher levels of alcohol consumption and gambling habits resulted in lower evaluation ratings; however this significant interaction was driven by alcohol consumption. 
In regards to the likelihood of recommending hiring job candidates, a main effect of alcohol consumption was found to be significant, $F(2,127)=87.66, p<.001$, Partial Eta Squared $=.58$. Gambling was not found to be significant, $F(1,128)=2.91, p>.05$, Partial Eta Squared $=.02$. The interaction between alcohol consumption and gambling was found to be significant, $F(2,127)=3.98, p<.05$, Partial Eta Squared $=.06$, however, this significant interaction was driven by alcohol consumption. A main effect of alcohol consumption was found to be significant. The higher job candidates' alcohol consumption levels, the lower participants rated job candidates. Exposure to higher levels of alcohol consumption resulted in lower evaluation ratings. The interaction between alcohol consumption and gambling habits was also found to be significant. Exposure to higher levels of alcohol consumption and gambling habits resulted in lower evaluation ratings; however this significant interaction was driven by alcohol consumption.

In regards to addressing job candidates' expected performance, a main effect of alcohol consumption was found to be significant, $F(2,124)=87.55, p<.001$, Partial Eta Squared $=.59$. Gambling was not found to be significant, $F(1,125)=1.82, p>.05$, Partial Eta Squared $=.01$. The interaction between alcohol consumption and gambling was not found to be significant, $F(2,124), p>.05$, Partial Eta Squared $=.001$. A main effect of alcohol consumption was found to be significant. The higher job candidates' alcohol consumption level, the lower participants rated job candidates. Exposure to higher levels of alcohol consumption resulted in lower evaluation ratings.

In regards to assessing the likelihood of job candidates quitting, a main effect of alcohol consumption was found to be significant, $F(2,125)=37.94, p<.001$, Partial Eta Squared $=.38$. Gambling was found to be significant, $F(1,126)=4.82, p<.05$, Partial Eta Squared $=.04$. The interaction between alcohol consumption and gambling was found to be significant, $F(2,125)=3.57, p<.05$, Partial Eta Squared $=.05$, however, this significant interaction was driven by alcohol consumption. A main effect of alcohol consumption was found to be significant. The higher job candidates' alcohol consumption level, the higher participants rated job candidates' likelihood for quitting. Exposure to higher levels of alcohol consumption resulted in higher evaluation ratings for job candidates quitting. Including gambling habits in general interests and on personal web page graphics also resulted in higher evaluation ratings of job candidates' likelihood for quitting. Exposure to gambling resulted in higher evaluation ratings for job candidates quitting. The interaction between alcohol consumption and gambling habits was also found to be significant. Exposure to higher levels of alcohol consumption and including gambling habits resulted in higher evaluation ratings for job candidates quitting; however this significant interaction was driven by alcohol consumption.

Alcohol consumption was found to be significant for all five questions. Gambling habits were only found to be significant for question five. The interaction between alcohol consumption and gambling habits were found to be significant for questions two, three, and five. 


\section{Discussion}

The purpose of this study was to evaluate the influence of information about applicants obtained via non-traditional sources on employer evaluations. Numerous lay sources indicate that information available via the internet is finding its way into employment related decision making but few empirical studies have evaluated the impact of this information on applicants and employers. In general, results support these lay observations and suggest that this "extra" information impacts applicant evaluations and has the potential to influence selection decisions.

In summary, alcohol consumption was found to significantly influence job candidates' evaluations for all five assessed areas (likelihood of extending an interview invitation, assessment of qualifications, recommendation for hire, expected work performance, and expected work tenure). Job candidates who included "no" or "some" levels of alcohol consumption on their personal web pages usually received similar evaluation ratings. Job candidates who included "a lot" level of alcohol consumption on their personal web pages received lower evaluation ratings. This results indicates that participants found the "no" or "some" levels of alcohol consumption more acceptable than the "a lot" level of alcohol consumption. Results suggested that high alcohol consumption had the most significant negative impact on job candidates' evaluations.

Gambling habits were found to affect job candidates' evaluations for one area that was measured in this study (expected work tenure). Participants who gambled received high evaluations for the likelihood of quitting the account administrator position. This results indicates that job candidates who did not gamble were expected to have longer work tenure than job candidates who gambled.

Interestingly, although results for gambling were not as extreme as those for alcohol consumption, information on gambling habits appeared to exacerbate the differences associated with different levels of alcohol consumption. The interaction between alcohol consumption and gambling habits was found to significantly affect job candidates' evaluations for three areas (assessment of qualifications, recommendation for hire, and expected work tenure). Results suggested that the interaction between alcohol consumption and gambling habits also had a significant negative impact on job candidates' evaluations; however it appears that the majority of these significant interactions were driven by alcohol consumption.

\section{Limitations and Future Research}

There are several limitations inherent in this study, consideration of which may enhance future research on this topic. One limitation of this study is that all participants were students at a single university located in the southern United States. An effort was made to collect data from employed students that were representative of the general population, but the fact remains that the sample was composed of mostly, female and Caucasian college students. Additionally, cultural norms regarding issues such as gambling and alcohol consumption vary and this may limit the applicability of the 
specific results of this study outside of the southern United States. Including a more diverse and balanced sample may enhance the generalizability of future research.

Names may contain alternative sources of information about candidates (O'Leary, Durham, Weathington, Cothran, \& Cunningham, 2009). Although an effort was made to use common names and all candidates were presented as white males, it is possible that some participants utilized the names provided to infer information about applicants. It is hoped that any effect of names on results would be consistent across participant evaluations of the same candidate in both the resume only and resume plus web page conditions but names remain an uncontrolled variable that could have confounded the study results.

The resumes themselves may also have been problematic. The high quality of all six resumes may have restricted the range of responses to the single-item measures of perceived recommendations to interview, ability, recommendations to hire, performance, and likelihood of quitting. Although a large percentage of the present sample had previous work experience, the participants were not recruiting professionals. Future research would be enhanced by sampling college recruiters in organizations related to human resources. Another extension of this research would be to measure the effects of other characteristics commonly reported on social networking websites. Future research should evaluate the influence of highly visible, hot-topic issues (e.g., sexual orientation, smoking, etc.) as well as more subtle differences (e.g., names, setting of profile photos, etc.).

\section{$\underline{\text { Practical Implications }}$}

This study suggests that information people post on personal web pages can impact the selection decision making process. Results suggest that exposure to negative or questionable personal information posted on web pages negatively impacts evaluations of job candidates. Therefore, it is especially important for individuals applying for a job, and especially recent graduates, to realize the impact information posted on social networking websites may have on the selection decision making process. As a general observation, based on the amount and type of information individuals voluntarily provide about themselves, it does not seem that most understand the potential impact of this information on their lives.

In conclusion, evidence suggests that the information being posted on social networking websites and obtainable via web searches is readily available to individuals making employment related decisions. Going beyond the debate about whether or not this information should be used, it can be argued that if it is available it will be used and negative information or information that may appear as "red flags" negatively affects potential job candidates' evaluations.

\section{References}

Arvey, R. D. (1979). Unfair discrimination in the employment interview: Legal and 
psychological aspects. Psychological Bulletin, 86(4), 736-765.

Barton, P. E. (2008, January-February). How many college graduates does the U.S. labor force really need? Change: The Magazine for Higher Learning. Retrieved from http:// http://www.changemag.org/Archives/Back Issues/January-February 2008.

Brown, B. K., \& Campion, M. A. (1994). Biodata phenomenology: Recruiters' perceptions and use of biographical information in resume screening. Journal of Applied Psychology, 79(6), 897-908.

Cole, M. S., Field, H. S., \& Giles, W. F. (2003). What can we uncover about applicants based on their resumes? A field study. Applied HRM Research, 8(2), 51-62.

Dipboye, R. L., Fontenelle, G. A. \& Garner, K. (1984). Effects of previewing the application on interview process and outcomes. Journal of Applied Psychology, 69(1), 118-128.

Finder, A. (2006, June 11). For some, online persona undermines a resume. The New York Times. Retrieved from http://www.nytimes.com.

Gatewood, R. D., \& Feild, H. S. (2001). Human Resource Selection (5 ${ }^{\text {th }}$ edition). Orlando, FL: Harcourt College Publishers.

Hargittai, E. (2008). Whose space? Differences among users and non-users of social network sites. Journal of Computer-Mediated Communication, 13(1), 276-297.

Hecker, D. E. (1992, July). Reconciling conflicting data on jobs for college graduates. Monthly Labor Review, 115(7), 3-12.

Hutchinson, K. L. (1984). Personnel administrators' preferences for resume content. Journal of Business Communication, 21(4), 5-14.

Levine, E. L., \& Flory, A. (1975). Evaluation of job applications - A conceptual framework. Public Personnel Management, 4, 378-385.

Mallory, A. (2007, December 15). Penn State student loses job over Tech victim costume. The Roanoke Times, Retrieved from http://www.roanoke.com.

Occupational Outlook Quarterly. (1994, Summer). The College labor market: Yesterday, today, and tomorrow, 3.

O'Leary, B. J., Durham, C. R., Weathington, B. L., Cothran, D. L., \& Cunningham, C. J. L. (2009). Racial Identity as a Moderator of the Relationship between Perceived Applicant Similarity and Hiring Decisions. The Journal of Black Psychology, 35(1), 63-77.

Pannone, R. (1994). Blue collar selection. In G. S. Stokes, M. D. Mumford, W. A. Owens (Eds.), Biodata handbook: Theory, research, and use of biographical information in selection and performance prediction (pp. 261-273). Palo Alto, CA: Consulting Psychologists Press, Inc.

Shelley, K. J. (July, 1992). The future of jobs for college graduates. Monthly Labor Review, 115(7), 13-21.

Shelley, K. J. (1994, Summer). More job openings-even more new entrants: The outlook for college graduates, 1992-2005. Occupational Outlook Quarterly, 5-9.

Thoms, P., McMaster, R., Roberts, M. R., \& Dombkowski, D. A. (1999). Resume characteristics as predictors of an invitation to interview. Journal of Business and Psychology, 13(3), 339-356. 